\title{
The novel SAM domain protein Aveugle is required for Raf activation in the Drosophila EGF receptor signaling pathway
}

\author{
Jean-Yves Roignant, Sophie Hamel, ${ }^{1}$ Florence Janody, ${ }^{2}$ and Jessica E. Treisman $^{3}$ \\ Skirball Institute for Biomolecular Medicine and Department of Cell Biology, New York University School of Medicine, \\ New York, New York 10016, USA
}

\begin{abstract}
Activation of the Raf kinase by GTP-bound Ras is a poorly understood step in receptor tyrosine kinase signaling pathways. One such pathway, the epidermal growth factor receptor (EGFR) pathway, is critical for cell differentiation, survival, and cell cycle regulation in many systems, including the Drosophila eye. We have identified a mutation in a novel gene, aveugle, based on its requirement for normal photoreceptor differentiation. The phenotypes of aveugle mutant cells in the eye and wing imaginal discs resemble those caused by reduction of EGFR pathway function. We show that aveugle is required between ras and raf for EGFR signaling in the eye and for mitogen-activated protein kinase phosphorylation in cell culture. aveugle encodes a small protein with a sterile $\alpha$ motif (SAM) domain that can physically interact with the scaffold protein connector enhancer of Ksr (Cnk). We propose that Aveugle acts together with Cnk to promote Raf activation, perhaps by recruiting an activating kinase.
\end{abstract}

[Keywords: Raf; MAP kinase; receptor tyrosine kinase; EGFR; photoreceptor; SAM]

Supplemental material is available at http://www.genesdev.org.

Received November 4, 2005; revised version accepted January 24, 2006.

Many receptor tyrosine kinases (RTKs), including the epidermal growth factor receptor (EGFR) and fibroblast growth factor receptor (FGFR), signal through the Ras/ mitogen-activated protein kinase (MAPK) pathway (Nishida and Gotoh 1993). These receptors have important developmental functions and are also misregulated in a variety of cancers (Holbro and Hynes 2004), making it critical to understand their signaling mechanism. Genetic screens in Drosophila and Caenorhabditis elegans, coupled with biochemical analysis in cultured cells, have allowed the identification of numerous Ras/MAPK pathway components (Rubin et al. 1997; Moghal and Sternberg 2003).

The Drosophila eye is a particularly useful system for genetic analysis of the EGFR pathway, which has welldefined functions in photoreceptor development (Freeman 1997; Halfar et al. 2001; Yang and Baker 2003). As the morphogen Hedgehog $(\mathrm{Hh})$ drives progression of the

Present addresses: ${ }^{1}$ Ecole Normale Supérieure, CNRS UMR8542, 46 rue d'Ulm, 75230 Paris Cedex 05, France; ${ }^{2}$ Institut de Biologie du Développement de Marseille, Campus de Luminy, Case 907, 13288 Marseille Cedex 9, France.

${ }^{3}$ Corresponding author.

E-MAIL treisman@saturn.med.nyu.edu; FAX (212) 263-7760.

Article and publication are at http://www.genesdev.org/cgi/doi/10.1101/ gad.1390506. morphogenetic furrow across the eye disc, it induces the expression of the transcription factor Atonal, which specifies the first photoreceptor to differentiate in each cluster, R8 (Jarman et al. 1995; Dominguez 1999). Once the R8 photoreceptor has been specified, it sequentially recruits additional photoreceptors, cone cells, and pigment cells from the surrounding pool of undifferentiated cells. The signal for this recruitment is the EGFR ligand Spitz (Spi), which is secreted by R8 and subsequently by other photoreceptors as they differentiate (Freeman 1996). Production of the downstream feedback inhibitor Argos (Aos), which binds to Spi and blocks its binding to the receptor (Klein et al. 2004), restricts the response to Spi to a small number of cells, allowing the stepwise recruitment of ommatidial cell types (Freeman 1997). A second RTK, Sevenless, also contributes to R7 differentiation (Freeman 1996). The precursors of the R2, R5, R3, and R4 photoreceptors remain arrested in the G1 phase of the cell cycle after leaving the morphogenetic furrow. This arrest also requires EGFR signaling, but occurs at a lower threshold than the differentiation response (Yang and Baker 2003). Mitosis of the remaining cells in the second mitotic wave is likewise driven by EGFR signaling, through activation of the target gene string, which encodes a cdc25 phosphatase (Baonza et al. 2002; Yang and Baker 2003). Finally, loss of EGFR signaling leads to 
apoptosis, as this pathway is required to down-regulate the expression and activity of the proapoptotic protein head involution defective (Hid) (Bergmann et al. 1998; Kurada and White 1998; Halfar et al. 2001; Yang and Baker 2003).

Ligand binding to the EGFR and other RTKs induces their dimerization and autophosphorylation (Schlessinger 2002). The pathway downstream has been elucidated by both genetic and biochemical analysis (for review, see Nishida and Gotoh 1993; Rubin et al. 1997; Moghal and Sternberg 2003). Phosphorylated EGFR can interact with the adaptor protein downstream of receptor kinases (Drk), which links it to son of sevenless (Sos), a guanine nucleotide exchange factor for Ras1. This stimulates conversion of Ras1 to an active GTP-bound form, while the reverse reaction leading to an inactive GDPbound form is enhanced by Gap1. In a complex scaffolded by connector enhancer of ksr (Cnk), GTP-Ras1 activates the kinase Raf. This initiates a kinase cascade in which Raf, in the presence of the scaffold protein kinase suppressor of ras (Ksr), phosphorylates downstream of raf1 (Dsor1 or MEK), which itself phosphorylates MAPK. Phosphorylated MAPK can enter the nucleus, where it phosphorylates two Ets transcription factors, Pointed P2 (PntP2) and Yan. EGFR target gene expression is activated by phosphorylated PntP2, while the transcriptional repressor Yan is exported to the cytoplasm and degraded upon phosphorylation. Two transcriptional targets of the pathway are another isoform of pointed, pntP1, and the feedback inhibitor aos (Gabay et al. 1996; Golembo et al. 1996).

One step in this pathway that remains unclear is the precise mechanism by which Ras activates Raf (Dhillon and Kolch 2002; Wellbrock et al. 2004). Raf activation in mammalian cells requires its recruitment to the plasma membrane through binding to Ras (Herrmann et al. 1995; Marais et al. 1995), as well as dephosphorylation by protein phosphatase $2 \mathrm{~A}$ of binding sites for the 14-3-3 protein in the N-terminal region of Raf (Jaumot and Hancock 2001; Kubicek et al. 2002; Dumaz and Marais 2003), and phosphorylation of sites upstream of the catalytic domain and within the activation segment by unidentified kinases (Fabian et al. 1993; Zhang and Guan 2000; Chong et al. 2001). In Drosophila, the scaffold protein Cnk appears to have dual roles in the activation event; its N-terminal region, which contains essential sterile $\alpha$ motif (SAM) and conserved region in Cnk (CRIC) domains, promotes Raf activation downstream of Ras, while its C-terminal region, which contains a Raf-binding domain, plays an inhibitory role downstream of Raf (Therrien et al. 1999; Douziech et al. 2003). It has been proposed that Src42 binding antagonizes this inhibition, allowing Cnk to integrate Ras and Src42 signals (Laberge et al. 2005). In C. elegans, Cnk-1 acts downstream of Raf dephosphorylation, but upstream of the activating phosphorylation events (Rocheleau et al. 2005). SAM domains have been shown to mediate homo- or heterooligomerization in both nuclear and membrane proteins (Stapleton et al. 1999; Kim et al. 2001, 2002; Ramachander et al. 2002), suggesting that the function of the
SAM domain of Cnk might be to interact with another SAM domain protein; however, such a partner has not yet been identified.

Using a genetic mosaic screen to identify genes required for normal photoreceptor differentiation, we have isolated a mutation in a novel Drosophila gene, aveugle (ave), with phenotypes characteristic of components of the EGFR signaling pathway in both the eye and wing discs. Loss of ave appears to reduce but not abolish signaling through the pathway, and epistasis tests in vivo and in cell culture indicate that ave acts between ras and raf to promote MAPK phosphorylation. ave encodes a small protein consisting almost entirely of a SAM domain. Ave can directly bind to Cnk in a SAM-domaindependent manner, and colocalizes and coimmunoprecipitates with Cnk in S2 cells. We suggest that the interaction between Ave and Cnk recruits an activator to Raf.

\section{Results}

aveugle is required for EGFR signaling during eye development

In a mosaic genetic screen for genes required for photoreceptor differentiation (Janody et al. 2004), we isolated one ethyl methanesulfonate (EMS)-induced lethal allele of a new gene that we have called aveugle (ave). In the wild-type eye disc, photoreceptors are recruited sequentially to clusters posterior to the morphogenetic furrow (Fig. 1A) and can be visualized by staining with the neuronal nuclear marker Elav (Fig. 1B,D). In ave mutant clones, fewer Elav-expressing nuclei were present within each cluster (Fig. 1E,G). However, expression of Senseless (Sens), a marker for the first photoreceptor to differentiate, R8, was largely normal in ave mutant clones (Fig. 1C,F). Hedgehog produced by more posterior cells induces R8 differentiation by activating expression of the transcription factor Atonal (Jarman et al. 1995; Dominguez 1999|, while recruitment of all the other cell types to the cluster is dependent on EGFR signaling (Freeman 1996). We used markers for different ommatidial cell types (Fig. 1A) to determine which were missing in ave mutants. Staining for the cone cell marker Cut (Blochlinger et al. 1993) revealed that very few cone cells differentiated in ave mutant clones (Fig. 2A-C). Expression of Bar, a marker for the R1 and R6 photoreceptors (Higashijima et al. 1992), was also almost absent from ave mutant clones (Fig. 2D-F). However, Spalt, which labels R3 and R4 in anterior regions of the eye disc (Domingos et al. 2004), was less affected, indicating that a reduced number of $\mathrm{R} 3$ and $\mathrm{R} 4$ cells were able to differentiate in ave mutant clones (Fig. 2G-I). ave mutant clones also contained Elav-expressing cells not labeled by Spalt, most likely corresponding to R2 and R5 cells. The requirement for ave in the differentiation of cells other than R8 suggested that ave might be involved in EGFR signaling during eye development. However, the ave mutant phenotype appeared less severe than mutations in other components of the EGFR pathway, such as cnk (Fig. 1H-J; Therrien et al. 1998). Most ave mutant 


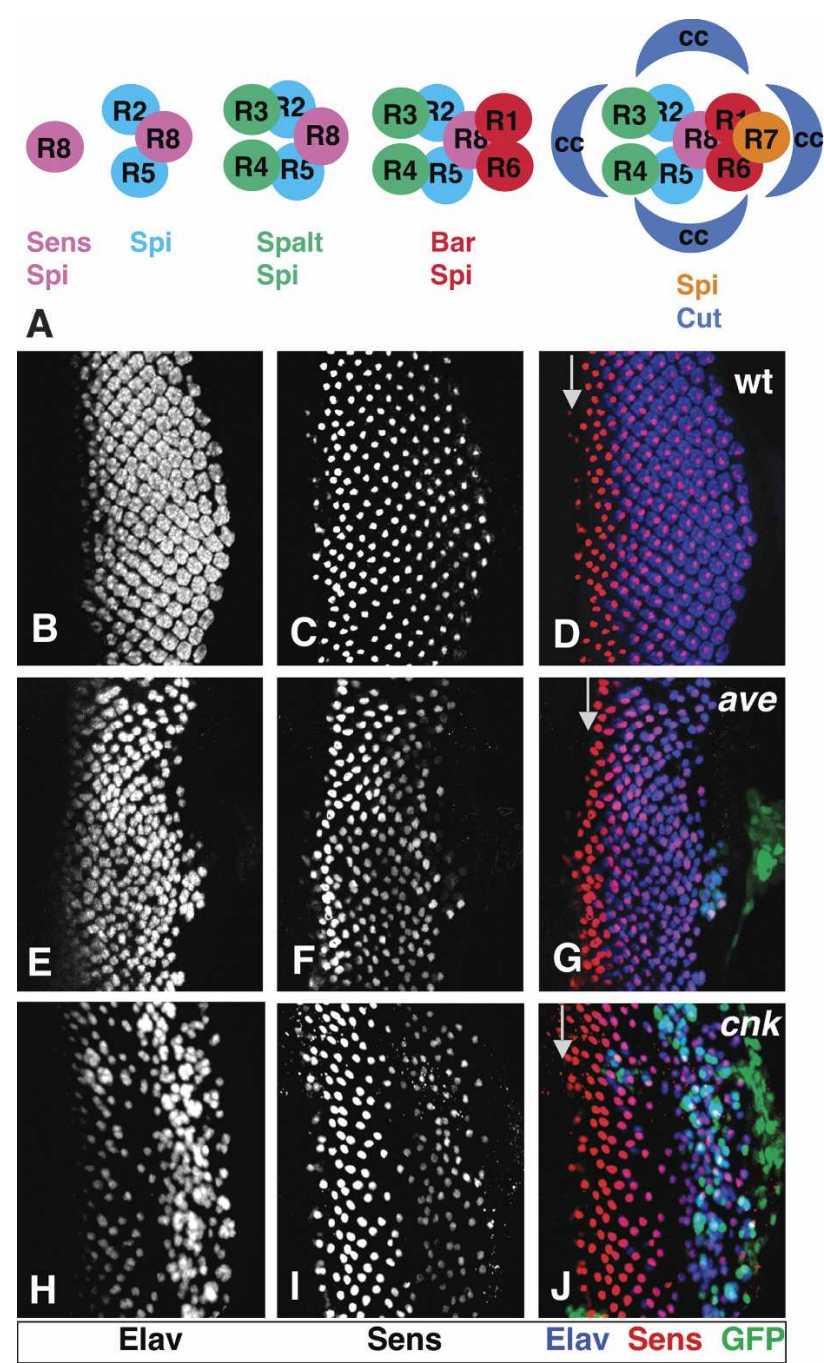

Figure 1. ave is required for photoreceptor differentiation. $(A)$ Diagram of the stages of photoreceptor recruitment in the larval eye disc. In the morphogenetic furrow (left), R8 differentiation is triggered by Hh. R8 then expresses Spi to recruit R2 and R5, followed by R3 and R4, R1 and R6, R7, and then the four cone cells. Expression of Spi in each cell as it differentiates contributes to the recruitment of subsequent cell types. The markers used in this study to recognize R8, R3/R4, R1/R6, and cone cells are indicated. $(B-J)$ Third instar eye imaginal discs with anterior to the left. The arrows in $D, G$, and $J$ indicate the morphogenetic furrow. $(B-D)$ Wild-type eye disc. $(E-G)$ Eye disc with a large ave mutant clone marked by lack of GFP (green in $G$ ). (H-J) Eye disc with a large cnk mutant clone marked by lack of GFP (green in $J)$. Photoreceptors are stained with anti-Elav $(B, E, H$; blue in $D, G, J)$, and $\mathrm{R} 8$ is stained with anti-Senseless $(C, F, I$; red in $D, G, J)$. Most cnk mutant ommatidia contain only R8, while most ave mutant ommatidia contain one or two photoreceptors in addition to $\mathrm{R} 8$.

clusters contained one or two photoreceptors in addition to R8, while most cnk mutant clusters contained only R8. Loss of R8 cells, probably due to cell death, was also more pronounced in cnk than in ave mutant clones (Fig. $1 \mathrm{~F}, \mathrm{I})$.

In addition to promoting photoreceptor differentia- tion, EGFR signaling is required for cell survival and for cell cycle regulation in the eye disc (Halfar et al. 2001; Yang and Baker 2003). We next tested whether these aspects of EGFR function required ave. EGFR signaling maintains G1 arrest of the precluster cells R2-R5, preventing them from accumulating Cyclin B protein, which is present in cells that have passed the G1/S checkpoint but not yet divided (Yang and Baker 2003). As shown in Figure 2, J-L, Cyclin B expression was observed in an increased number of cells posterior to the morphogenetic furrow in ave mutant clones, indicating a partial failure of G1 arrest. However, some cells that did not express Elav were still able to degrade Cyclin B (Fig. 2L). Activated Caspase 3, a marker for apoptotic cells (Srinivasan et al. 1998), was present in a small subset of cells in ave mutant clones, suggesting that some additional cell death occurs in the absence of ave (Fig. 2MO). Based on its relatively weak effects on photoreceptor differentiation, cell cycle arrest, and apoptosis, we hypothesize that loss of ave in the eye reduces but does not abolish EGFR signaling.

To support this conclusion, we looked at the expression of a direct target gene of the EGFR signaling pathway in the eye. Expression of the transcription factor PntP1 is directly induced upon activation of the pathway (Gabay et al. 1996). In wild-type eye discs, PntP1 is strongly expressed in groups of cells within the morphogenetic furrow. Consistent with reduced EGFR signaling in ave mutant cells, we found an autonomous decrease, but not complete loss, of PntP1 expression (Fig. 2P-R). Taken together, these findings demonstrate that ave is required for normal levels of EGFR signaling during eye development.

ave is required for EGFR activity in wing development

We next examined the effects of loss of ave on EGFR signaling outside the eye. EGFR signaling is required for wing vein formation, and activates expression of the target gene aos along the vein primordia in the wing disc (Gabay et al. 1997; Guichard et al. 1999). Induction of ave mutant clones led to a loss of wing veins in the adult wing (Fig. 3B). Consistent with the lack of vein differentiation, aos-lacZ expression was autonomously lost from ave mutant clones in the wing disc (Fig. 3F,G). EGFR signaling has also been shown to define the notum primordium of the wing disc, marked by expression of Homothorax (Hth) (Azpiazu and Morata 2000; Wang et al. 2000; Zecca and Struhl 2002). Homozygous ave mutants can survive until the third larval instar; at this stage, we found that their wing discs had greatly reduced notum primordia (Fig. 3D).

\section{ave encodes a novel SAM domain protein}

We used complementation tests with the deficiency kit available from the Bloomington Drosophila stock center as well as recombination mapping relative to $\mathrm{P}\left(\mathrm{w}^{+}\right)$elements to map ave to chromosome region 51C1-C5 (Fig. 
Roignant et al.
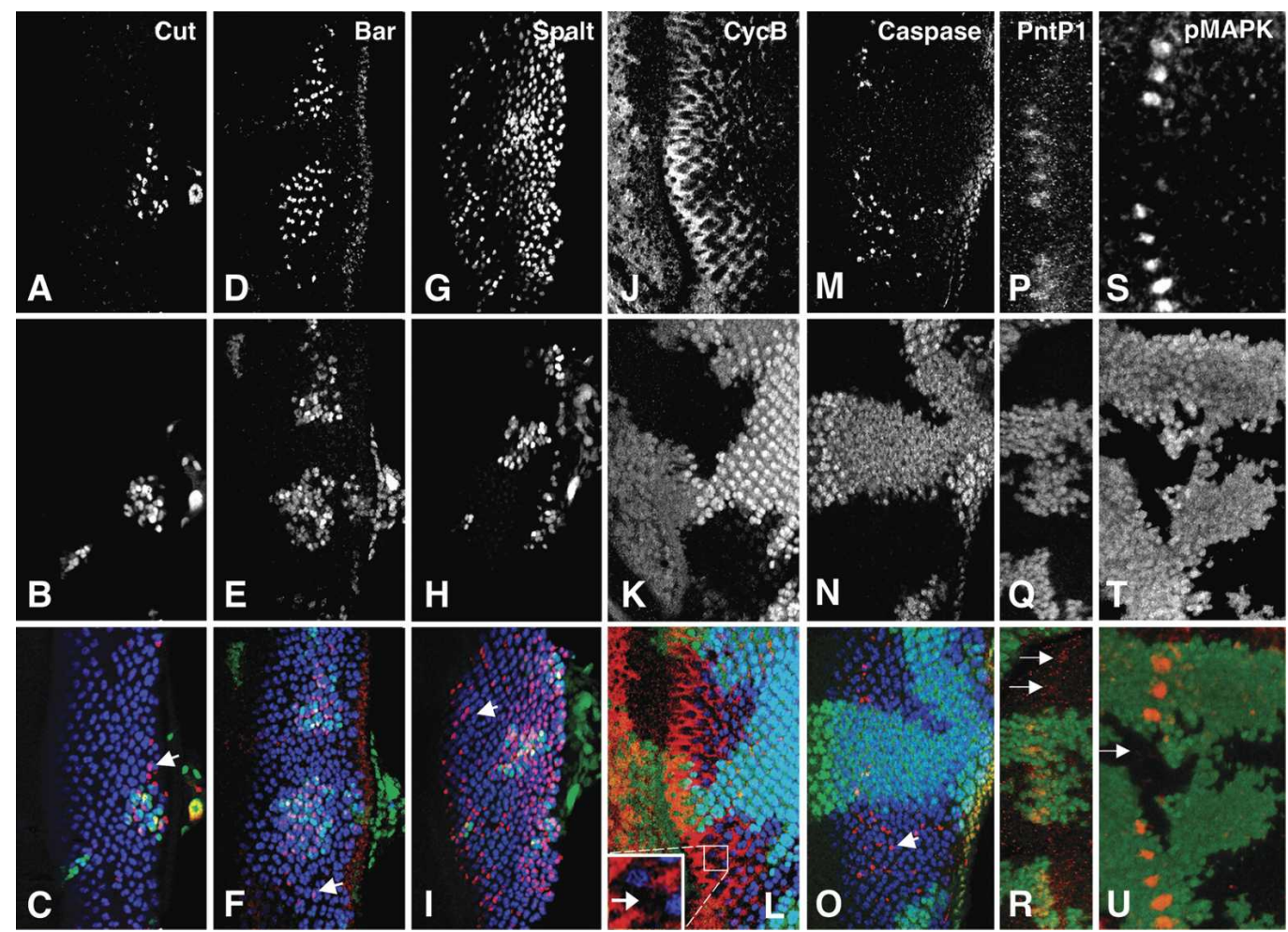

Figure 2. ave is required for EGFR signaling during eye development. ave mutant clones generated in third instar eye discs are marked by loss of GFP $(B, E, H, K, N, Q, T$; green in $C, F, I, L, O, R, U) .(A-C)$ Cone cells were stained with anti-Cut $(A$, red in $C)$ and photoreceptors with anti-Elav (blue in $C)$. Very few cone cells differentiate in ave mutant clones. $(D-F) \mathrm{R} 1$ and R6 photoreceptors were stained with anti-Bar $(D$, red in $F)$ and all photoreceptors with anti-Elav (blue in $F)$. Most ave mutant clusters lack R1 and R6 photoreceptors. (G-I) R3 and R4 photoreceptors were labeled with anti-Spalt $(G$, red in $I)$ and all photoreceptors with anti-Elav (blue in $I)$. Some ave mutant cells express Spalt, indicating that some R3 and R4 photoreceptors can differentiate in the absence of Ave. In the posterior of the eye disc, Spalt stains R7, R8, and cone cells. The arrows in $C, F$, and $I$ indicate ave mutant cells that do express Cut, Bar, and Spalt respectively. $(J-L)$ Staining for Cyclin B $(J$, red in $L$ ) and Elav (blue in $L$ ). Cyclin B expression is increased in ave mutant clones, indicating that more cells reenter the cell cycle posterior to the morphogenetic furrow. However, some cells that do not differentiate as photoreceptors also do not express Cyclin B (arrow in magnification in $L$ ). ( $M-O)$ Cell death was monitored with an antibody to activated Caspase $(M$, red in $O)$. An increase in activated Caspase staining was observed in ave mutant cells posterior to the morphogenetic furrow (arrow), indicating that some cells undergo apoptosis. $(P-R)$ Expression of PntP1 $(P$, red in $R)$, a positive target of EGFR signaling, is reduced in ave mutant cells but not completely lost (arrows). ( $S-U$ ) Activation of MAPK was assessed with an antibody to phosphorylated MAPK ( $S$, red in $U$ ). Phospho-MAPK staining is autonomously missing in ave mutant cells (arrow).

4A). Since we found only a single allele of ave in a screen in which multiple alleles of genes of moderate size were obtained (Janody et al. 2004), we expected ave to be a small gene. We therefore sequenced the six smallest predicted genes contained in this region from ave homozygous larvae, and found a nonsense mutation in the predicted gene CG30476 that would truncate the encoded protein at amino acid 34 (Fig. 4B). This mutation was not present in the isogenic parental flies used for the screen. We also found that ave failed to complement the deficiency $D f(R p n 6)^{2 F}$, which completely removes CG30476 and partially deletes the adjacent genes Rpn6 and CG10151 (Fig. 4A). Finally, we generated transgenic fly lines expressing an HA-tagged full-length CG30476 protein under the control of UAS sequences. Expression of this transgene in ave mutant clones was sufficient to rescue the photoreceptor differentiation defect (Fig. 5C,D). Overexpressing CG30476 ubiquitously throughout development using daughterless-GAL4 had no ap- parent effect on wild-type flies. Based on the stop codon present in the mutant allele, the failure to complement $D f(R p n 6)^{2 F}$, and the ability of CG30476 cDNA to rescue the ave mutant phenotype, we conclude that ave corresponds to the predicted gene CG30476.

ave encodes a small protein of 106 amino acids that contains a SAM domain (Fig. 4B). This domain is a protein module $\sim 70$ residues long that has been implicated in protein-protein interactions (Stapleton et al. 1999; Kim et al. 2001, 2002; Ramachander et al. 2002). Our allele of ave is likely to be a functional null, as the truncated protein would contain only 11 amino acids of the SAM domain. Homologs of ave exist in other species, including humans (Fig. 4B), but their functions are still unknown. Using in situ hybridization, we found that ave transcripts were present ubiquitously throughout development (Fig. 4C), as might be expected for a component required for transduction of a common developmental signal. 


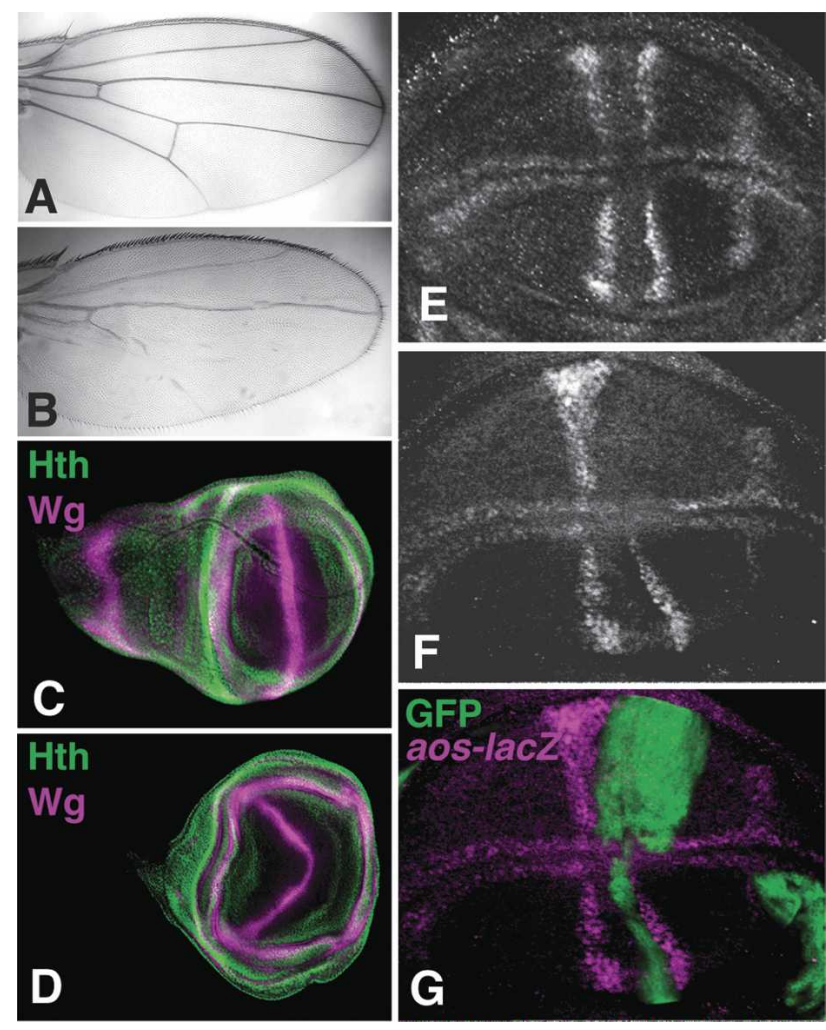

Figure 3. ave is required for EGFR signaling in wing development. (A) Wild-type adult wing. (B) Wing containing large unmarked ave mutant clones. Wing vein formation is disrupted. $(C)$ Wild-type wing disc. $(D)$ Homozygous ave mutant wing disc. $\mathrm{Wg}$ is stained in magenta and Homothorax in green. The wing pouch of homozygous ave mutant discs is largely normal in size, but most of the notum is missing. $(E-G)$ Third instar wing discs stained with anti- $\beta$-galactosidase reflecting argos-lac $Z$ expression $(E, F$, magenta in $G)$. $(E)$ Wild type. $(F, G)$ Wing disc with ave mutant clone positively marked by GFP expression (green in $G$ ). argos is not expressed in vein primordia in the absence of ave.

\section{Ave functions downstream of Ras but upstream of Raf}

To determine the point at which Ave acts in the EGFR signaling pathway, we attempted to rescue the photoreceptor differentiation defect in ave mutant clones by expressing activated forms of components of the pathway. $\mathrm{Ras}^{\mathrm{V} 12}$ is a constitutively activated form of Drosophila Ras1, and Raff ${ }^{F 179}$ encodes a gain-of-function version of Raf in which the N-terminal domain of Raf is deleted. Expressing either of these gain-of-function alleles in wild-type eye discs caused ectopic photoreceptor differentiation (Fig. 5E,F; data not shown). ave mutant clones expressing Ras ${ }^{\mathrm{V} 12}$ displayed the photoreceptor differentiation defect typical of ave mutant clones (Fig. 5G,H). In contrast, $R a f^{F 179}$ expressed in ave mutant clones was still able to induce excessive photoreceptor differentiation (Fig. 5I,J). These results strongly suggest that Ave acts downstream of Ras but upstream of Raf in the EGFR signaling pathway that controls photoreceptor differentiation. In this case, ave should be required for down- stream events such as MAPK activation. We tested this prediction using an antibody directed specifically against phosphorylated MAPK (Gabay et al. 1997). PhosphoMAPK is present at high levels in the group of cells that strongly express PntP1 within the morphogenetic furrow. As expected, based on our epistasis experiments, we found that phospho-MAPK was lost from ave mutant clones (Fig. 2S-U). Together, these results confirm that Ave acts upstream of the Raf/MEK/MAPK kinase cascade in the eye disc.

We next examined whether the requirement for Ave at this position in the EGFR pathway is conserved in other cell types. The embryonically derived Drosophila S2 cell line expresses most components of the EGFR pathway (Roy et al. 2002). We first showed by RT-PCR that ave is also endogenously expressed in these cells and that it could be strongly depleted by RNA interference (RNAi) (Supplementary Fig. 1A). To test the requirement for Ave in RAS/MAPK signaling, we removed endogenous Ave by RNAi in S2 cells expressing Ras ${ }^{\mathrm{V} 12}$ and used MAPK phosphorylation as a readout of pathway activation. As shown in Figure 5K, RNAi directed against Ras or against the downstream components Cnk or Ksr specifically blocked MAPK phosphorylation (lanes 5-7). Ave depletion gave an identical result (Fig. 5K, lane 8), indicating that Ave is required downstream of Ras for MAPK activation in S2 cells. We next examined the effect of removing Ave on MAPK activation induced by an activated form of Raf (Tor ${ }^{4021} \mathrm{RAFc}$ ) (Douziech et al. 2003); activated Ras and activated Raf induced equivalent levels of MAPK phosphorylation (data not shown). As predicted by the epistasis experiments in photoreceptors, Ave was not required for MAPK phosphorylation induced by activated Raf (Fig. 5L, lane 5). Cnk depletion likewise had no effect; as a control, we showed that depletion of MEK, the kinase directly downstream of Raf, abrogated MAPK activation (Fig. 5L, lanes 4,6). These results indicate that Ave acts between Ras and Raf in S2 cells, suggesting a general requirement for Ave at this position in the EGFR signaling pathway.

\section{Ave colocalizes and interacts with Cnk}

Cnk is a scaffolding protein that is required downstream of Ras for Raf activation and, like Ave, contains a SAM domain. This prompted us to ask whether Cnk and Ave might function in the same complex. We first determined whether Ave and Cnk showed the same subcellular localization. Flag-tagged Cnk has been shown to be predominantly located in the cytoplasm of S2 cells, with some accumulation at the plasma membrane (Therrien et al. 1998). We cotransfected adherent S2R+ cells with Flag-Cnk, HA-tagged Ave, and a nuclear form of GFP. As expected, Cnk was found in the cytoplasm with some enrichment at the plasma membrane (Fig. 6B). Ave and Cnk clearly colocalized in both locations (Fig. 6A-C), supporting a possible interaction between these two proteins. We confirmed this interaction using a yeast twohybrid assay. Ave strongly interacted with the $\mathrm{N}$-terminal domain of Cnk, consisting of the SAM, CRIC, and 
Roignant et al.

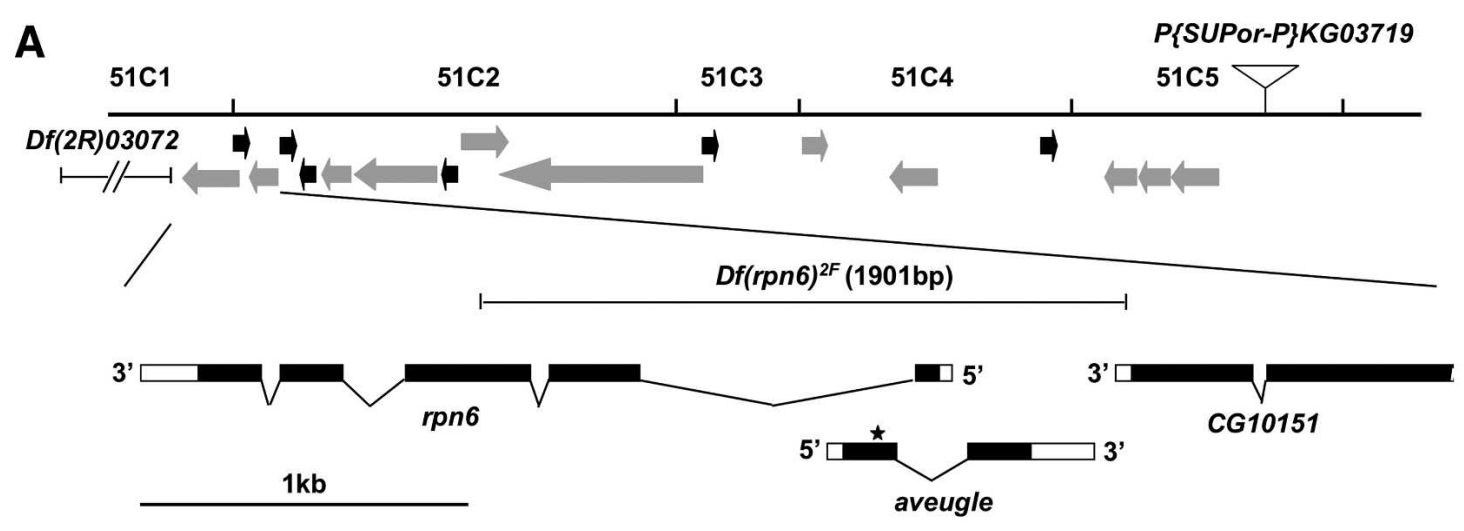

B
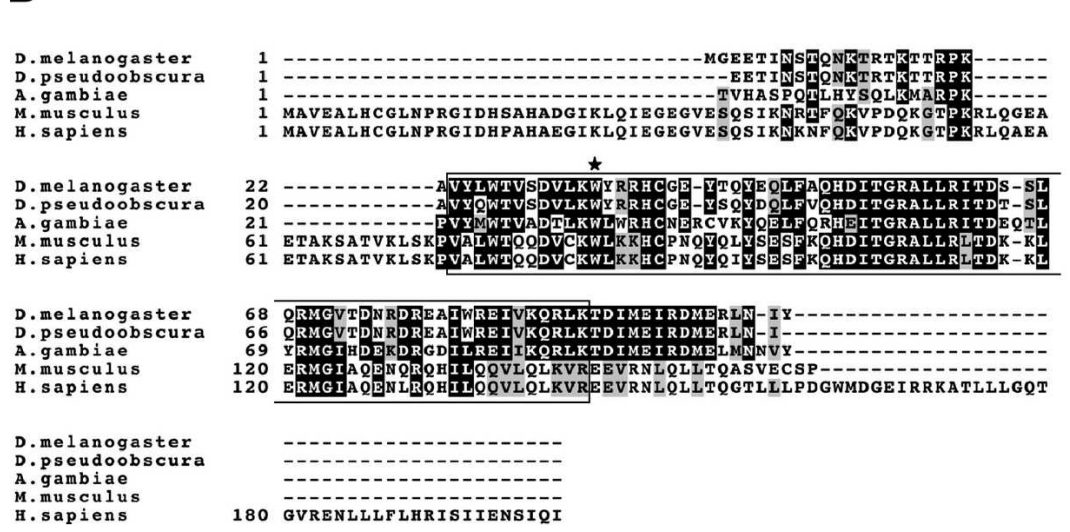

C

Figure 4. ave encodes a novel SAM domain protein. (A) Diagram of the ave gene. The top line represents the genomic DNA. aveugle was mapped to 51C1-51C5, distal to $D f(2 R) 03072$ and proximal to the P element $P\{S U P o r-P\} K G 03719$. Of the 17 genes enclosed in this region (represented by arrows), the six smallest were sequenced (black arrows). A molecular defect was found in the first exon of the predicted gene CG30476 (see asterisk) that we named aveugle. Closed and open boxes correspond to the coding and noncoding sequences, respectively. The deficiency $D f(R p n 6)^{2 F}$ that entirely removes CG30476 and partially deletes the rpn6 and CG10151 genes does not complement our ave allele. (B) Amino acid sequence comparison of the predicted homologs of ave. The alignment was generated using CLUSTALW. Identical residues are highlighted in black and similar residues in gray. The GenBank accession numbers are: Homo sapiens (NP 997,389), Drosophila pseudoobscura (EAL25193), Anopheles gambiae (XP 320,359). The position of the stop codon in $a v e^{108 V}$ at Trp34 is indicated with an asterisk. The SAM domain is boxed. $(C)$ In situ hybridization of embryos or eye-antennal imaginal discs (bottom) with antisense (left) or sense ave probes (right). ave RNA is ubiquitously expressed throughout embryogenesis and in the eye-antennal disc.

PDZ domains (Fig. 6D). Deletion of the SAM domain from this Cnk construct abolished the interaction with Ave (Fig. 6D), suggesting that the interaction is mediated by SAM domain heterodimerization. We did not detect any direct interaction between Ave and either the Nterminal or C-terminal domains of Raf; as a control, we confirmed that the C-terminal domain of Raf could interact with MEK (Fig. 6D; Therrien et al. 1996). Ave was also unable to homodimerize (Fig. 6D). To determine whether Ave also interacts with Cnk in vivo, cell lysates from S2R+ cells expressing Flag-Cnk and HA-Ave were immunoprecipitated with anti-HA antibody and probed with anti-Cnk antibody. Cnk was coimmunoprecipitated with HA-Ave, and the association was specific since no Cnk was immunoprecipitated from cells not transfected with HA-Ave (Fig. 6D). Since Cnk and Ave act at the same position in the pathway, share a SAM domain, colocalize within the cell, and physically interact, it is likely that they function together to promote Raf activation.

\section{Discussion}

Although Raf activation is a critical step in RTK signaling pathways, its mechanism is still not fully understood. Key steps include Raf translocation to the plasma membrane and release of its protein kinase domain from an intramolecular inhibitory domain through changes in the phosphorylation state of specific residues. These processes occur in the context of the essential scaffolding proteins Cnk and Ksr. Here we identify the novel SAM domain protein Ave as another component required for Raf activation. Ave is required between Ras and Raf for EGFR signaling in differentiating photoreceptors and in S2 cells, and is present in the same complex as Cnk. We discuss below how Ave may contribute to Raf activation.

ave is required for maximal levels of EGFR signaling

Loss of ave in the eye disc disrupts normal photoreceptor differentiation; while R8 cells differentiate correctly, most of the other photoreceptors are missing. Although 

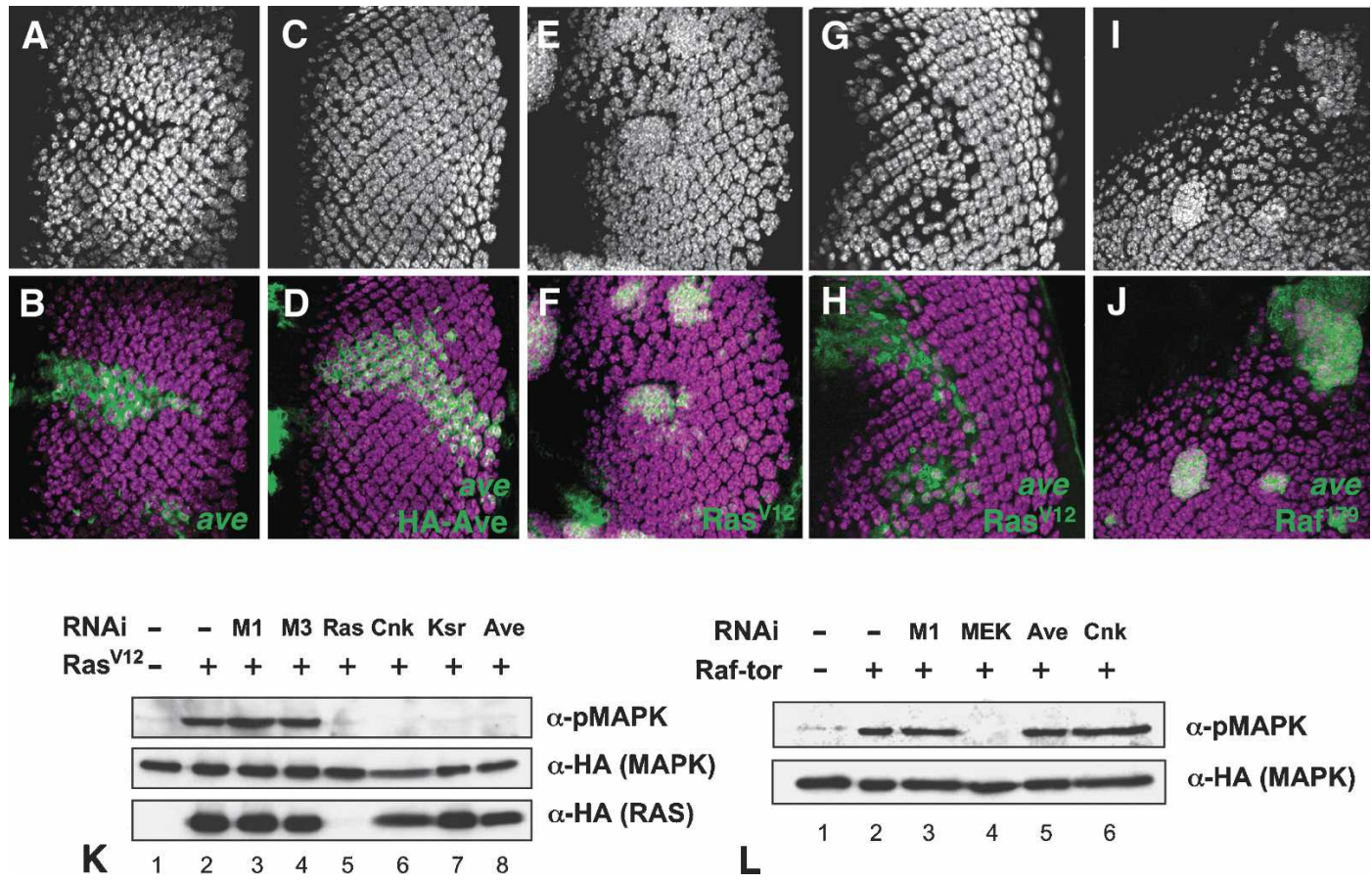

Figure 5. ave acts downstream of Ras but upstream of Raf. $(A-I)$ Eye discs in which photoreceptors are stained with anti-Elav $(A, C, E, G, I$; magenta in $B, D, F, H, J)$. Clones mutant for ave and/or expressing UAS transgenes are labeled by GFP expression (green in $B, D, F, H, J) .(A, B)$ Photoreceptors are missing from ave mutant clones. $(C, D)$ Expression of UAS-HA-ave in ave mutant clones fully rescues photoreceptor differentiation. $(E, F)$ Expression of UAS-Ras ${ }^{V 12}$ in wild-type clones leads to excessive photoreceptor differentiation. $(G, H)$ Expression of UAS-Ras ${ }^{V 12}$ does not rescue the defect observed in ave mutant clones. $(I, J)$ Expression of UAS-Raf $f^{F 179}$ induces excessive photoreceptor differentiation in ave mutant clones. (K) S2 cells transfected with HA-MAPK alone (lane 1) or with HA-MAPK and Ras ${ }^{\mathrm{V} 12}$ incubated alone (lane 2) or with $15 \mu \mathrm{g}$ of the indicated dsRNAs (lanes 3-8). M1 and M3 were used as negative controls and correspond to dsRNA directed against two regions of the mago nashi gene. Western blots are shown for phospho-MAPK, HA-MAPK, and HA-Ras. (L) S2 cells transfected with HA-MAPK alone (lane 1), with HA-MAPK and Tor ${ }^{4021}$ RAFc incubated alone (lane 2), or with $15 \mu \mathrm{g}$ of the indicated dsRNAs (lanes 3-6). Like Cnk, Ave is required for MAPK phosphorylation induced by activated Ras, but not for MAPK phosphorylation induced by activated Raf. MEK is a positive control required downstream of activated Raf.

the mutation we isolated is likely to be a null allele of ave, its phenotype is weaker than loss of function of core components of the EGFR pathway, including cnk. R8 is still able to recruit a few photoreceptors in the absence of ave, and only a small proportion of ave mutant cells die during the third larval instar. The reduced expression in ave mutant cells of PntP1, a direct target of the pathway, suggests that ave is required to increase the overall level of EGFR signaling. We note that MAPK phosphorylation is undetectable in the absence of ave in both eye disc cells and S2 cells, suggesting that examination of EGFR responses in vivo is more sensitive than detection of phospho-MAPK.

If loss of ave simply reduces the level of EGFR signaling, it would imply that distinct thresholds of EGFR signaling recruit different subclasses of ommatidial cells, since ave has a stronger effect on recruitment of R1, R6, and cone cells than on R2-R5. The dependence of many different ommatidial cell fates on EGFR signaling has been taken to imply that the response of an undifferentiated cell to the EGFR signal changes over time (Freeman 1996). This change in cellular competence may be due to changes in transcription factor expression in signal-receiving cells (Flores et al. 2000; Xu et al. 2000). The intermediate phenotype of ave mutants suggests that specification of early differentiating photoreceptors such as R3 and R4 requires a lower level of EGFR signaling than specification of later differentiating cells such as R1, R6, and cone cells. Interestingly, phosphorylated MAPK levels are lower in the region of the eye disc in which R2-R5 differentiate than in more posterior regions (Yang and Baker 2003). In addition, R7 differentiation has been shown to require both EGFR and Sevenless to signal through the Ras/MAPK module, suggesting that an elevated amount of signal is required for its specification (Freeman 1996). An alternative means of temporal control is the induction by EGFR activity of signaling molecules required to recruit later cell types; for instance, EGFR recruits cone cells in part by activating expression in photoreceptors of the Notch ligand Delta (Tsuda et al. 2002). ave might be required for the expression of specific EGFR target genes such as Delta that promote sequential induction of late-differentiating cell types.

In addition to photoreceptor differentiation, EGFR signaling in the eye is required for cell survival and cell cycle arrest; these two functions have been proposed to require a lower level of EGFR activity than differentiation of R1-R7 (Halfar et al. 2001; Yang and Baker 2003). 

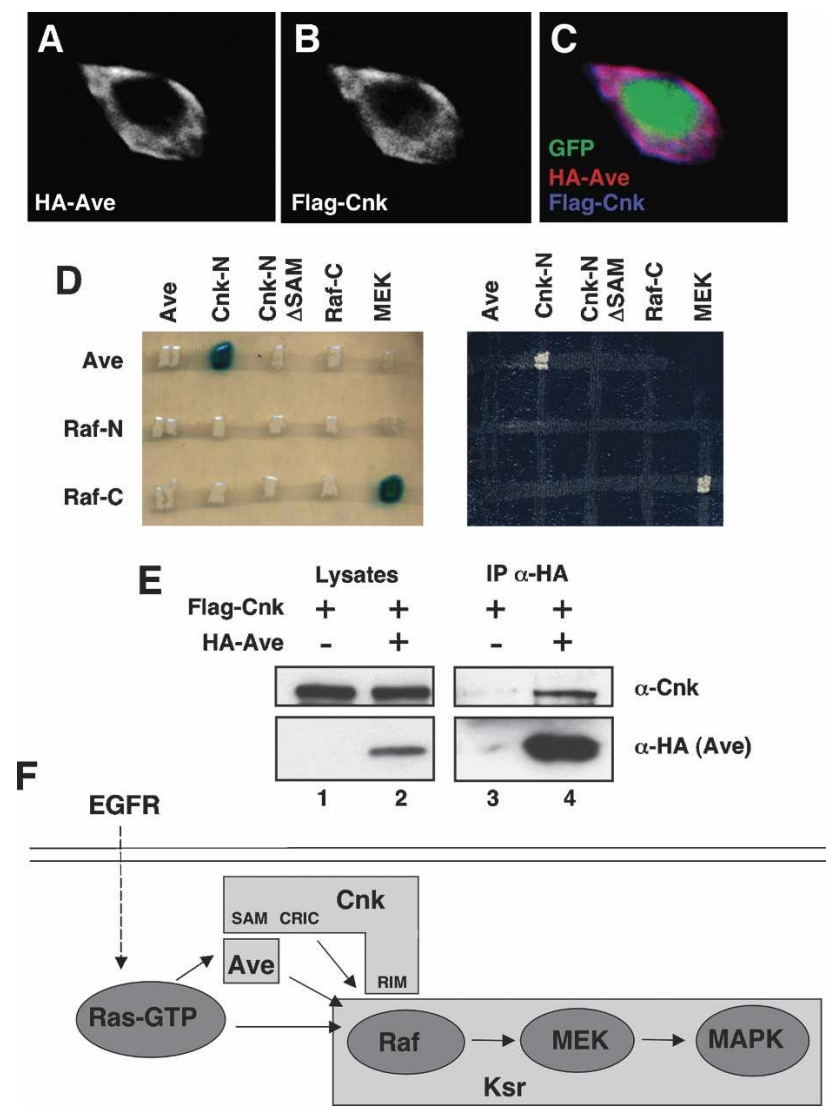

Figure 6. Ave colocalizes and interacts with Cnk in S2R+cells. $(A-C)$ Subcellular localization of Cnk and Ave in S2R+cells. Cells were cotransfected with pMet-Flag-Cnk, pUAST-HA-Ave, pUAST-GFP, and actin-GAL4, and immunostained with anti$\mathrm{HA}(A$, red in $C)$ and anti-Flag $(B$, blue in $C) .(C) \mathrm{GFP}$ is in green. Cnk and Ave display an identical subcellular localization. $(D)$ Interaction of Ave and Cnk in a yeast two-hybrid assay. Each patch corresponds to diploid yeast colonies coexpressing LexA DNA-binding domain fusion proteins (labeled on left) with B42 transcriptional activation domain fusion proteins (labeled on top). Diploids were monitored for $\beta$-galactosidase activity (left) and for growth on Leu-selective medium (right). The positive interactions are galactose inducible (not shown). Interaction of MEK with the C-terminal domain of Raf was included as a control. Ave directly interacts with the $\mathrm{N}$-terminal domain of Cnk, and this interaction requires the SAM domain of Cnk. (E) Coimmunoprecipitation of Flag-Cnk with HA-Ave. Extracts from S2R+ cells expressing Flag-Cnk either alone (lane 3) or with HA-Ave (lane 4) were immunoprecipitated using HA beads. Expression of Ave and Cnk was monitored by Western blotting with anti-HA and anti-Cnk antibodies, respectively. Lanes 1 and 2 show 2\% of the input. (F) Model for Ave function in the Ras/MAPK module. The SAM and CRIC domains of Cnk are critical to integrate the Ras signal and enhance Raf catalytic function. Our results suggest that Ave binds to Cnk, probably through the SAM domain, and relays the Ras signal to promote Raf activation (see Discussion). In the absence of Ave, Cnk still has some activity, possibly provided by the positive function of the CRIC domain.

Our results support this conclusion, since we found that some ave mutant cells that do not differentiate as photoreceptors are still able to arrest in G1. However, we found an increase in apoptosis in ave mutant clones, despite their ability to differentiate some photoreceptors in addition to $\mathrm{R} 8$. This result suggests that there may not be a sharp threshold between the differentiation and survival responses; the level of EGFR signaling achieved in the absence of ave can allow differentiation of some photoreceptors without preventing all apoptosis.

The requirement for Ave in other EGFR-dependent processes appears to be variable. In the wing disc, ave is essential for notum growth and for expression of the EGFR target gene aos; aos is likely to be a high-threshold target, as it is expressed in cells containing high levels of phosphorylated MAPK (Gabay et al. 1997). However, ave is not required for all signaling by EGFR or the RTK Torso during embryogenesis. Embryos lacking both the maternal and zygotic contribution of ave did not show any detectable change in midline aos-lacZ or terminal tailless expression (data not shown). As in the wing disc, aos is thought to be activated by high levels of EGFR signaling, due to its overlap with phospho-MAPK staining (Gabay et al. 1997). ave might be redundant with another molecule expressed at this stage of development, although no close sequence homolog is present in the Drosophila genome. Alternatively, the Ras/MAPK module may use a distinct mechanism for signal transduction during embryogenesis. In this regard, it will be interesting to test whether $c n k$ is required for EGFR signaling in the embryo.

\section{Ave interacts with Cnk to promote Raf activation}

Genetic and biochemical studies have shown that the scaffolding protein Cnk is required for RTK signaling downstream of Ras but upstream of Raf (Therrien et al. 1998; Douziech et al. 2003). Its N-terminal SAM and CRIC domains are essential for its function in promoting Raf activity (Douziech et al. 2003). SAM domains frequently act as homo- or heterodimerization motifs. The SAM domains of Ave and Cnk can directly interact in yeast, suggesting that the essential function of the SAM domain of Cnk may be to interact with Ave (Fig. 6F).

How might the interaction of Ave with Cnk promote Raf activation? Since Cnk binds to Raf through a C-terminal Raf-interacting motif (RIM) (Therrien et al. 1998), this binding is unlikely to require Ave. In addition, the RIM is dispensable for the transduction of Ras signaling and, in fact, seems to have an inhibitory effect on Ras signaling (Douziech et al. 2003). We have not observed any change in the strength of the interaction between Raf and Cnk when ave is removed by RNAi (Supplementary Fig. 1A,B). A more likely possibility is that association of Ave with Cnk helps to bring an activator kinase into proximity with Raf. Raf activation in mammalian cells involves dephosphorylation of inhibitory sites followed by phosphorylation of activating sites (for review, see Dhillon and Kolch 2002; Chong et al. 2003). However, the identity of the activating kinases is still unclear; Ksr was a candidate, but the current view is that it acts as a scaffolding protein rather than an active kinase (Morrison 2001). In C. elegans, epistasis tests suggest 
that Cnk promotes Raf activation after dephosphorylation but before the activating phosphorylation events (Rocheleau et al. 2005), consistent with a model in which Cnk in association with Ave attracts an activator kinase to Raf. Certain SAM domains have been shown to act as kinase-docking sites; for example, the SAM domain of ETS-1 provides a docking site for the ERK-2 MAPK, promoting phosphorylation of and transcriptional activation by ETS-1 (Seidel and Graves 2002). Likewise, the ETS-2 SAM domain serves as a docking site for the Cdc2 family kinase Cdk10 (Kasten and Giordano 2001). A search for other binding partners of Ave may lead to the identification of the activating kinase for Raf.

An alternative possibility is that association of Ave with Cnk could help to recruit Raf to the plasma membrane. In S2 cells, Cnk is required for membrane recruitment of Raf (Anselmo et al. 2002), but it may not be sufficient for this function, since overexpression in $\mathrm{CHO}$ cells of MAGUIN-1, the closest mammalian homolog of Drosophila Cnk, does not recruit Raf-1 to the plasma membrane (Yao et al. 2000). The SAM domain of human p73 has been shown to directly bind lipid membranes (Barrera et al. 2003), suggesting the possibility that Ave links Cnk or Raf directly to the plasma membrane. However, we have not seen a clear change in the subcellular localization of tagged Cnk when Ave is knocked down by RNAi (Supplementary Fig. 1C-F).

Another well-described property of SAM domains is their ability to polymerize, promoting the formation of homo- or hetero-oligomers. This mechanism underlies long-range transcriptional repression by the SAM domain proteins TEL and Polyhomeotic (Kim et al. 2001, 2002). In the context of Raf activation, it is possible that polymerization of Ave, together with Cnk and perhaps other SAM domain-containing proteins, leads to the formation of large scaffolding complexes in which the local concentration of Raf and/or its activators is increased. Interestingly, the yeast adaptor protein Ste50, which is required for the activation of a MAPKKK, Ste11 (Ramezani-Rad 2003), induces polymerization of Ste11 through interactions between the SAM domains of the two molecules (Bhattacharjya et al. 2005). This may stabilize a complex in which the Ste20 kinase can phosphorylate Ste11 (Ramezani-Rad 2003). A stabilizing function might explain why ave is not essential in all contexts in Drosophila, as high concentrations of the molecules it recruits could lead to Ave-independent signaling. The evolutionary conservation of Ave suggests that it is likely to regulate the Ras/Raf/MAPK module in other species.

\section{Materials and methods}

Fly stocks, genetics, and molecular biology

A single ave allele $\left(a v e^{108 V}\right)$ was isolated from a mosaic screen for mutations affecting photoreceptor differentiation (Janody et al. 2004). This mutation failed to complement $D f(R p n 6)^{2 F}$ (Lier and Paululat 2002). The fly stocks used were da-GAL4, $\operatorname{argos}^{w 1}$, $\mathrm{UAS}$ ras $^{\text {V12 }}$ (FlyBase), UAS-rafF179 (Martin-Blanco et al. 1999), and $c n k^{14 C}$ (Janody et al. 2004). ave $e^{108 \mathrm{~V}}$ mutant clones were generated by crossing FRT42D, ave/CyO, $\mathrm{y}^{+}$males to FRT42D, ubi-GFP; hsFLP122 females. Large clones were generated by crossing FRT42D, ave/CyO, $\mathrm{y}^{+}$males to FRT42D, ubi-GFP, $M(2) 58 F / C y O, y^{+}$; eyFLP1 females, or to FRT42D, ubi-GFP, $\mathrm{M}(2) 58 \mathrm{~F} / \mathrm{CyO}, \mathrm{y}^{+}$; hsFLP122 females. Epistasis experiments were done using the MARCM system. For example, Ras ${ }^{\mathrm{V} 12}$ was overexpressed in ave mutant clones by crossing FRT42D, ave; UAS-ras ${ }^{V 12} /$ SM6-TM6B males to eyFLP1, UAS-GFP; FRT42D, tub-GAL80; tub-GAL4/TM6B females. Germline clones were made by crossing hsFLP122; FRT42D, ubi-GFP to FRT42D, ave/ CyO. Larvae were heat shocked for $1 \mathrm{~h}$ at $38.5^{\circ} \mathrm{C}$ in both first and second instar. The resulting hsFLP122; FRT42D, ave/ FRT42D, ubi-GFP females were crossed to FRT42D, ave; argoslacZ/SM6-TM6B males, and embryos were selected for the absence of GFP staining.

The coding regions of CG30476, CG12859, CG10153, CG12854, CG12856, and CG10205 were amplified by PCR from genomic DNA obtained from homozygous ave mutant first instar larvae, and the PCR products were sequenced. The fulllength ave coding region was amplified by PCR from the RE67675 clone (Drosophila Genomics Resource Center) using Pfu Turbo and cloned into HA-pUAST as an EcoRI/XhoI fragment to generate UAS-HA-ave. Transgenic flies were generated by standard methods.

PCR fragments encoding full-length Ave, the Raf N-terminal domain (amino acids 1-418), and the Raf C-terminal domain (amino acids 419-739) were subcloned into the bait vector pEG202 (Gyuris et al. 1993). PCR fragments corresponding to full-length Ave, the Cnk N-terminal domain (amino acids 1-378), the Cnk N-terminal domain without the SAM domain (amino acids 86-378), the Raf C-terminal domain (amino acids 419-739), and full-length MEK were subcloned into the prey vector pJG4-5 (Gyuris et al. 1993). All constructs were confirmed by sequencing.

\section{Immunostaining, histology, and in situ hybridization}

The primary antibodies used were rat anti-Elav (1:5; Developmental Studies Hybridoma Bank), guinea pig anti-Sens (1:1000) (Frankfort et al. 2001), rabbit anti-BarS12 (1:50) (Higashijima et al. 1992), mouse anti-cut 2B10 (1:1) (Blochlinger et al. 1993), mouse anti-Cyclin B 1:20; Developmental Studies Hybridoma Bank), rabbit anti-CM1 (1:500; BD Pharmingen), rabbit antiSalm (1:100) (Domingos et al. 2004), mouse anti-Wingless (1:20; Developmental Studies Hybridoma Bank), rabbit anti-PntP1 (1:500) (Alvarez et al. 2003), mouse anti-dpERK (phosphoMAPK; 1:50; Sigma), rabbit anti-GFP (1:2500; Molecular Probes), mouse anti-GFP (1:2500; Roche), mouse anti- $\beta$-galactosidase (1:200; Promega), rabbit anti-Homothorax (1:500) (Kurant et al. 1998), rat anti-HA 3F10 (1:1000; Roche), and rabbit antiFlag (1:500; Sigma). TSA enhancement was used for the antidpERK antibody. Eye and wing imaginal discs were dissected in $0.1 \mathrm{M}$ sodium phosphate buffer ( $\mathrm{pH}$ 7.2) and then fixed in PEM (0.1 M PIPES at $\mathrm{pH} 7,2 \mathrm{mM} \mathrm{MgSO}{ }_{4}, 1 \mathrm{mM}$ EGTA) containing $4 \%$ formaldehyde. Washes were done in $0.1 \mathrm{M}$ phosphate buffer with $0.2 \%$ Triton X-100. Appropriate fluorescent-conjugated secondary antibodies were used (1:200; Jackson Immunoresearch Laboratories). Fluorescent images were collected on a Leica TCS NT confocal microscope. S2R+ cells were fixed in PBS containing $4 \%$ formaldehyde and stained with appropriate antibodies. Adult wings were mounted in methyl salicylate: Canada balsam (1:2). Digoxigenin-UTP-labeled RNA probes homologous to the ave coding region were used for in situ hybridization. Embryos were hybridized as described (Lehmann and Tautz 1994), and eye discs were hybridized as described (Bach et al. 2003). 


\section{Cell culture}

S2 and S2R+ cells were maintained in Schneider's medium (GIBCO) supplemented with $10 \%$ fetal calf serum and $50 \mathrm{U}$ of penicillin-streptomycin (GIBCO). Cells were transfected with Effectene (QIAGEN) according to the manufacturer's instructions. pMet FL-CNK, pMet-HARas ${ }^{\mathrm{V} 12}$, pMet-pyoTor ${ }^{4021}$ RAFc, and pMet-HAMAPK constructs were kindly provided by Marc Therrien (Universite de Montreal, Montreal, Quebec, Canada) (Douziech et al. 2003). UAS plasmids were cotransfected with actin-GAL4. Expression of Ras ${ }^{\mathrm{V} 12}$ and other copper-inducible constructs was induced $48 \mathrm{~h}$ post-transfection by adding 0.7 $\mathrm{mM} \mathrm{CuSO}_{4}$ to the cell medium. Cells were harvested either 18 $\mathrm{h}$ (epistasis experiments) or $48 \mathrm{~h}$ (coimmunoprecipitation experiments) after induction and lysed in ice-cold buffer $150 \mathrm{mM}$ Tris- $\mathrm{HCl}$ at $\mathrm{pH}$ 7.5, $150 \mathrm{mM} \mathrm{NaCl}, 1 \% \mathrm{NP}-40,2 \mathrm{mM}$ EDTA, 1 mM PMSF, $1 \mathrm{mM} \mathrm{NaVO}_{4}, 200 \mu \mathrm{m}$ each Aprotinin, Leupeptin, Pepstatin [epistasis experiments] or $20 \mathrm{mM}$ Tris- $\mathrm{HCl}$ at $\mathrm{pH} 8$, $100 \mathrm{mM} \mathrm{NaCl}, 0.5 \%$ NP-40, 10\% glycerol, 2 mM EDTA, $1 \mathrm{mM}$ PMSF, $1 \mathrm{mM} \mathrm{NaVO}$, $200 \mu \mathrm{m}$ each Aprotinin, Leupeptin, Pepstatin [coimmunoprecipitation experiments]).

\section{Immunoprecipitation and Western blotting}

Immunoprecipitations were performed by incubating cell lysates with protein G-agarose or protein A-agarose for $1 \mathrm{~h}$ at $4^{\circ} \mathrm{C}$. Beads were then pelleted and lysates were transferred to a new tube; $50 \mu \mathrm{L}$ of anti-HA Affinity Matrix (Roche) or $4 \mu \mathrm{L}$ of antiRaf antibody (gift from Deborah Morrison, National Cancer Institute-Frederick, Frederick, MD) were added to the lysate and incubated at $4^{\circ} \mathrm{C}$ overnight. Protein A-agarose was added for $2 \mathrm{~h}$ for the Raf immunoprecipitation experiment. Beads were pelleted and washed five times with IP buffer, $20 \mu \mathrm{L}$ of $3 \times$ reducing sample buffer were added, and the samples were boiled for 10 min, centrifuged, and loaded onto SDS-PAGE gels. Gels were transferred to a nitrocellulose membrane (Bio-Rad) and were blocked overnight with TBST (TBS $+0.1 \%$ Tween 20 ) supplemented with $5 \%$ low-fat milk. Membranes were incubated with TBST with $5 \%$ milk supplemented with antibodies for $2 \mathrm{~h}$ at room temperature. Blots were washed with TBST for $30 \mathrm{~min}$ and incubated with HRP-conjugated secondary antibodies (1:5000; Jackson Laboratories or Amersham) for another hour. Blots were developed with ECL chemiluminescence (Pierce). The antibodies used were mouse anti-HA (1:1000; Covance), mouse anti-Cnk (Douziech et al. 2003), and mouse anti-dpERK (1:2500; Sigma).

\section{Two-hybrid assays}

The yeast strain EGY48 containing the lacZ reporter plasmid pSH18-34 was transformed with a pEG202 derivative containing the LexA DNA-binding domain sequence fused in frame to either the Ave, Raf-N, or Raf-C coding sequences. The yeast strain RFY206 was transformed with a pJG4-5 derivative containing the B42 activation domain sequence fused in frame with Ave, Cnk-N, Cnk-NASAM, RAF-C, or MEK. Expression of all proteins in yeast was confirmed by Western blotting. EGY48 and RFY206 transformants were mated, and diploids were assayed for growth on selective medium and for $\beta$-galactosidase activity (Gyuris et al. 1993).

\section{RNAi experiments}

Double-stranded RNAs (dsRNAs) were generated using the MEGAscript T7 kit (Ambion). DNA fragments (between 0.5 and $1 \mathrm{~kb}$ ) containing sequences for the targeted proteins were am- plified by PCR. Each PCR primer contained at its $5^{\prime}$ end the T7 RNA polymerase-binding site (GAATTAATACGACTCAC TATAGGGAGA). PCR products were purified using the QIAquick PCR purification kit (Qiagen). One microgram of DNA was used per in vitro transcription reaction. RNA was precipitated at $-20^{\circ} \mathrm{C}$, dried, and resuspended in $50 \mu \mathrm{L}$ of DEPC-treated $\mathrm{H}_{2} \mathrm{O}$. RNA strands were annealed by heating to $65^{\circ} \mathrm{C}$ for $30 \mathrm{~min}$ and then slowly cooling to room temperature. Fifteen micrograms of dsRNA were added to the cells $\left(10^{6}\right.$ cells plated per well of six-well tissue culture dishes) $2 \mathrm{~d}$ before transfection. Five-hundred nanograms of dsRNA were mixed with the DNA constructs during transfection. Another $15 \mu \mathrm{g}$ of dsRNA were added $1 \mathrm{~d}$ post-transfection.

\section{$R T-P C R$}

Total RNA was extracted from S2 cells using Trizol (Invitrogen). RT-PCR was performed using the Invitrogen SuperScript First-Strand kit in accordance with the manufacturer's instructions. Three micrograms of total RNA were used per reaction. The PCR primers used for $\beta$-actin were 5'-GCCGGTTA CTCTTTCACCACCA-3', 3'-GCGATCCAGACAGAGTACT TGC-5' and for ave, 5' -CGGTGAATACACCCAGTATG-3', 3'TTAAATTTAATCTAGAATTTTGCGCTC-5'.

\section{Acknowledgments}

We thank Erika Bach, Hugo Bellen, Ulrike Gaul, Felix Karim, Rui Gonçalo Martinho, Bertrand Mollereau, Deborah Morrison, Achim Paululat, Adi Salzberg, Jim Skeath, Marc Therrien, the Bloomington Drosophila Stock Center, the Drosophila Genomics Resource Center, and the Developmental Studies Hybridoma Bank for fly stocks and reagents. The manuscript was improved by the critical comments of Erika Bach, Inés Carrera, Claude Desplan, Reza Farajian, Kerstin Hofmeyer, Nina Leeds, and Grant Miura. This work was supported by the National Institutes of Health (grant EY13777 to J.E.T.). J.-Y.R. was the recipient of a fellowship from the Fondation pour la Recherche Médicale.

\section{References}

Alvarez, A.D., Shi, W., Wilson, B.A., and Skeath, J.B. 2003. pannier and pointedP2 act sequentially to regulate Drosophila heart development. Development 130: 3015-3026.

Anselmo, A.N., Bumeister, R., Thomas, J.M., and White, M.A. 2002. Critical contribution of linker proteins to Raf kinase activation. J. Biol. Chem. 277: 5940-5943.

Azpiazu, N. and Morata, G. 2000. Function and regulation of homothorax in the wing imaginal disc of Drosophila. Development 127: 2685-2693.

Bach, E.A., Vincent, S., Zeidler, M.P., and Perrimon, N. 2003. A sensitized genetic screen to identify novel regulators and components of the Drosophila janus kinase/signal transducer and activator of transcription pathway. Genetics 165: 1149-1166.

Baonza, A., Murawsky, C.M., Travers, A.A., and Freeman, M. 2002. Pointed and Tramtrack69 establish an EGFR-dependent transcriptional switch to regulate mitosis. Nat. Cell Biol. 4: 976-980.

Barrera, F.N., Poveda, J.A., Gonzalez-Ros, J.M., and Neira, J.L. 2003. Binding of the C-terminal sterile $\alpha$ motif (SAM) domain of human p73 to lipid membranes. J. Biol. Chem. 278: 46878-46885. 
Bergmann, A., Agapite, J., McCall, K., and Steller, H. 1998. The Drosophila gene hid is a direct molecular target of Ras-dependent survival signaling. Cell 95: 331-341.

Bhattacharjya, S., Xu, P., Chakrapani, M., Johnston, L., and Ni, F. 2005. Polymerization of the SAM domain of MAPKKK Ste11 from the budding yeast: Implications for efficient signaling through the MAPK cascades. Protein Sci. 14: 828-835.

Blochlinger, K., Jan, L.Y., and Jan, Y.N. 1993. Postembryonic patterns of expression of cut, a locus regulating sensory organ identity in Drosophila. Development 117: 441-450.

Chong, H., Lee, J., and Guan, K.L. 2001. Positive and negative regulation of Raf kinase activity and function by phosphorylation. $E M B O$ J. 20: 3716-3727.

Chong, H., Vikis, H.G., and Guan, K.L. 2003. Mechanisms of regulating the Raf kinase family. Cell. Signal. 15: 463-469.

Dhillon, A.S. and Kolch, W. 2002. Untying the regulation of the Raf-1 kinase. Arch. Biochem. Biophys. 404: 3-9.

Domingos, P.M., Brown, S., Barrio, R., Ratnakumar, K., Frankfort, B.J., Mardon, G., Steller, H., and Mollereau, B. 2004. Regulation of R7 and R8 differentiation by the spalt genes. Dev. Biol. 273: 121-133.

Dominguez, M. 1999. Dual role for hedgehog in the regulation of the proneural gene atonal during ommatidia development. Development 126: 2345-2353.

Douziech, M., Roy, F., Laberge, G., Lefrancois, M., Armengod, A.V., and Therrien, M. 2003. Bimodal regulation of RAF by CNK in Drosophila. EMBO J. 22: 5068-5078.

Dumaz, N. and Marais, R. 2003. Protein kinase A blocks Raf-1 activity by stimulating 14-3-3 binding and blocking Raf-1 interaction with Ras. J. Biol. Chem. 278: 29819-29823.

Fabian, J.R., Daar, I.O., and Morrison, D.K. 1993. Critical tyrosine residues regulate the enzymatic and biological activity of Raf-1 kinase. Mol. Cell. Biol. 13: 7170-7179.

Flores, G.V., Duan, H., Yan, H., Nagaraj, R., Fu, W., Zou, Y., Noll, M., and Banerjee, U. 2000. Combinatorial signaling in the specification of unique cell fates. Cell 103: 75-85.

Frankfort, B.J., Nolo, R., Zhang, Z., Bellen, H., and Mardon, G. 2001. senseless repression of rough is required for R8 photoreceptor differentiation in the developing Drosophila eye. Neuron 32: 403-414.

Freeman, M. 1996. Reiterative use of the EGF receptor triggers differentiation of all cell types in the Drosophila eye. Cell 87: 651-660.

1997. Cell determination strategies in the Drosophila eye. Development 124: 261-270.

Gabay, L., Scholz, H., Golembo, M., Klaes, A., Shilo, B.Z., and Klambt, C. 1996. EGF receptor signaling induces pointed P1 transcription and inactivates Yan protein in the Drosophila embryonic ventral ectoderm. Development 122: 3355-3362.

Gabay, L., Seger, R., and Shilo, B.Z. 1997. In situ activation pattern of Drosophila EGF receptor pathway during development. Science 277: 1103-1106.

Golembo, M., Schweitzer, R., Freeman, M., and Shilo, B.Z. 1996. argos transcription is induced by the Drosophila EGF receptor pathway to form an inhibitory feedback loop. Development 122: 223-230.

Guichard, A., Biehs, B., Sturtevant, M.A., Wickline, L., Chacko, J., Howard, K., and Bier, E. 1999. rhomboid and Star interact synergistically to promote EGFR/MAPK signaling during Drosophila wing vein development. Development 126: 2663-2676.

Gyuris, J., Golemis, E.A., Chertkov, H., and Brent, R. 1993. Cdil, a human G1 and S phase protein phosphatase that associates with Cdk2. Cell 75: 791-803.

Halfar, K., Rommel, C., Stocker, H., and Hafen, E. 2001. Ras controls growth, survival and differentiation in the Dro- sophila eye by different thresholds of MAP kinase activity. Development 128: 1687-1696.

Herrmann, C., Martin, G.A., and Wittinghofer, A. 1995. Quantitative analysis of the complex between $\mathrm{p} 21$ ras and the Rasbinding domain of the human Raf-1 protein kinase. J. Biol. Chem. 270: 2901-2905.

Higashijima, S.-I., Kojima, T., Michiue, T., Ishimaru, S., Emori, Y., and Saigo, K. 1992. Dual Bar homeo box genes of Drosophila required in two photoreceptor cells, R1 and R6, and primary pigment cells for normal eye development. Genes \& Dev. 6: 50-60.

Holbro, T. and Hynes, N.E. 2004. ErbB receptors: Directing key signaling networks throughout life. Annu. Rev. Pharmacol. Toxicol. 44: 195-217.

Janody, F., Lee, J.D., Jahren, N., Hazelett, D.J., Benlali, A., Miura, G.I., Draskovic, I., and Treisman, J.E. 2004. A mosaic genetic screen reveals distinct roles for trithorax and Polycomb group genes in Drosophila eye development. Genetics 166: $187-200$.

Jarman, A.P., Sun, Y., Jan, L.Y., and Jan, Y.N. 1995. Role of the proneural gene, atonal, in formation of Drosophila chordotonal organs and photoreceptors. Development 121: 20192030.

Jaumot, M. and Hancock, J.F. 2001. Protein phosphatases 1 and 2A promote Raf- 1 activation by regulating 14-3-3 interactions. Oncogene 20: 3949-3958.

Kasten, M. and Giordano, A. 2001. Cdk10, a Cdc2-related kinase, associates with the Ets2 transcription factor and modulates its transactivation activity. Oncogene 20: 1832-1838.

Kim, C.A., Phillips, M.L., Kim, W., Gingery, M., Tran, H.H., Robinson, M.A., Faham, S., and Bowie, J.U. 2001. Polymerization of the SAM domain of TEL in leukemogenesis and transcriptional repression. EMBO J. 20: 4173-4182.

Kim, C.A., Gingery, M., Pilpa, R.M., and Bowie, J.U. 2002. The SAM domain of polyhomeotic forms a helical polymer. Nat. Struct. Biol. 9: 453-457.

Klein, D.E., Nappi, V.M., Reeves, G.T., Shvartsman, S.Y., and Lemmon, M.A. 2004. Argos inhibits epidermal growth factor receptor signalling by ligand sequestration. Nature 430: 1040-1044.

Kubicek, M., Pacher, M., Abraham, D., Podar, K., Eulitz, M., and Baccarini, M. 2002. Dephosphorylation of Ser-259 regulates Raf-1 membrane association. J. Biol. Chem. 277: 7913-7919.

Kurada, P. and White, K. 1998. Ras promotes cell survival in Drosophila by downregulating hid expression. Cell 95: 319329.

Kurant, E., Pai, C.Y., Sharf, R., Halachmi, N., Sun, Y.H., and Salzberg, A. 1998. Dorsotonals/homothorax, the Drosophila homologue of meis1, interacts with extradenticle in patterning of the embryonic PNS. Development 125: 1037-1048.

Laberge, G., Douziech, M., and Therrien, M. 2005. Src42 binding activity regulates Drosophila RAF by a novel CNK-dependent derepression mechanism. EMBO J. 24: 487-498.

Lehmann, R. and Tautz, D. 1994. In situ hybridization to RNA. Methods Cell Biol. 44: 575-598.

Lier, S. and Paululat, A. 2002. The proteasome regulatory particle subunit Rpn6 is required for Drosophila development and interacts physically with signalosome subunit Alien/ CSN2. Gene 298: 109-119.

Marais, R., Light, Y., Paterson, H.F., and Marshall, C.J. 1995 Ras recruits Raf- 1 to the plasma membrane for activation by tyrosine phosphorylation. EMBO T. 14: 3136-3145.

Martin-Blanco, E., Roch, F., Noll, E., Baonza, A., Duffy, J.B., and Perrimon, N. 1999. A temporal switch in DER signaling controls the specification and differentiation of veins and interveins in the Drosophila wing. Development 126: 5739-5747. 
Moghal, N. and Sternberg, P.W. 2003. The epidermal growth factor system in Caenorhabditis elegans. Exp. Cell Res. 284: 150-159.

Morrison, D.K. 2001. KSR: A MAPK scaffold of the Ras pathway? J. Cell Sci. 114: 1609-1612.

Nishida, E. and Gotoh, Y. 1993. The MAP kinase cascade is essential for diverse signal transduction pathways. Trends Biochem. Sci. 18: 128-131.

Ramachander, R., Kim, C.A., Phillips, M.L., Mackereth, C.D., Thanos, C.D., McIntosh, L.P., and Bowie, J.U. 2002. Oligomerization-dependent association of the SAM domains from Schizosaccharomyces pombe Byr2 and Ste4. J. Biol. Chem. 277: 39585-39593.

Ramezani-Rad, M. 2003. The role of adaptor protein Ste50-dependent regulation of the MAPKKK Ste11 in multiple signalling pathways of yeast. Curr. Genet. 43: 161-170.

Rocheleau, C.E., Ronnlund, A., Tuck, S., and Sundaram, M.V. 2005. Caenorhabditis elegans CNK-1 promotes Raf activation but is not essential for Ras/Raf signaling. Proc. Natl. Acad. Sci. 102: 11757-11762.

Roy, F., Laberge, G., Douziech, M., Ferland-McCollough, D., and Therrien, M. 2002. KSR is a scaffold required for activation of the ERK/MAPK module. Genes \& Dev. 16: 427-438.

Rubin, G.M., Chang, H.C., Karim, F., Laverty, T., Michaud, N.R., Morrison, D.K., Rebay, I., Tang, A., Therrien, M., and Wassarman, D.A. 1997. Signal transduction downstream from Ras in Drosophila. Cold Spring Harb. Symp. Quant. Biol. 62: 347-352.

Schlessinger, J. 2002. Ligand-induced, receptor-mediated dimerization and activation of EGF receptor. Cell 110: 669-672.

Seidel, J.J. and Graves, B.J. 2002. An ERK2 docking site in the Pointed domain distinguishes a subset of ETS transcription factors. Genes \& Dev. 16: 127-137.

Srinivasan, A., Roth, K.A., Sayers, R.O., Shindler, K.S., Wong, A.M., Fritz, L.C., and Tomaselli, K.J. 1998. In situ immunodetection of activated caspase- 3 in apoptotic neurons in the developing nervous system. Cell Death Differ. 5: 1004-1016.

Stapleton, D., Balan, I., Pawson, T., and Sicheri, F. 1999. The crystal structure of an Eph receptor SAM domain reveals a mechanism for modular dimerization. Nat. Struct. Biol. 6: 44-49.

Therrien, M., Michaud, N.R., Rubin, G.M., and Morrison, D.K. 1996. KSR modulates signal propagation within the MAPK cascade. Genes \& Dev. 10: 2684-2695.

Therrien, M., Wong, A.M., and Rubin, G.M. 1998. CNK, a RAFbinding multidomain protein required for RAS signaling. Cell 95: 343-353.

Therrien, M., Wong, A.M., Kwan, E., and Rubin, G.M. 1999. Functional analysis of CNK in RAS signaling. Proc. Natl. Acad. Sci. 96: 13259-13263.

Tsuda, L., Nagaraj, R., Zipursky, S.L., and Banerjee, U. 2002. An EGFR/Ebi/Sno pathway promotes Delta expression by inactivating $\mathrm{Su}(\mathrm{H}) / \mathrm{SMRTER}$ repression during inductive Notch signaling. Cell 110: 625-637.

Wang, S.H., Simcox, A., and Campbell, G. 2000. Dual role for Drosophila epidermal growth factor receptor signaling in early wing disc development. Genes \& Dev. 14: 2271-2276.

Wellbrock, C., Karasarides, M., and Marais, R. 2004. The RAF proteins take centre stage. Nat. Rev. Mol. Cell Biol. 5: 875885.

Xu, C., Kauffmann, R.C., Zhang, J., Kladny, S., and Carthew, R.W. 2000. Overlapping activators and repressors delimit transcriptional response to receptor tyrosine kinase signals in the Drosophila eye. Cell 103: 87-97.

Yang, L. and Baker, N.E. 2003. Cell cycle withdrawal, progression, and cell survival regulation by EGFR and its effectors in the differentiating Drosophila eye. Dev. Cell 4: 359-369.

Yao, I., Ohtsuka, T., Kawabe, H., Matsuura, Y., Takai, Y., and Hata, Y. 2000. Association of membrane-associated guanylate kinase-interacting protein-1 with Raf-1. Biochem. Biophys. Res. Commun. 270: 538-542.

Zecca, M. and Struhl, G. 2002. Subdivision of the Drosophila wing imaginal disc by EGFR-mediated signaling. Development 129: 1357-1368.

Zhang, B.H. and Guan, K.L. 2000. Activation of B-Raf kinase requires phosphorylation of the conserved residues Thr598 and Ser601. EMBO J. 19: 5429-5439. 


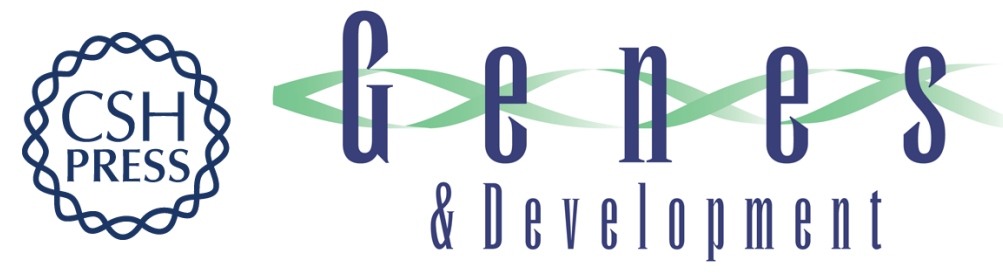

\section{The novel SAM domain protein Aveugle is required for Raf activation in the Drosophila EGF receptor signaling pathway}

Jean-Yves Roignant, Sophie Hamel, Florence Janody, et al.

Genes Dev. 2006, 20:

Access the most recent version at doi:10.1101/gad.1390506

Supplemental http://genesdev.cshlp.org/content/suppl/2006/03/16/20.7.795.DC1
Material

References This article cites 68 articles, 36 of which can be accessed free at:

http://genesdev.cshlp.org/content/20/7/795.full.html\#ref-list-1

License

Email Alerting Receive free email alerts when new articles cite this article - sign up in the box at the top

Service right corner of the article or click here.

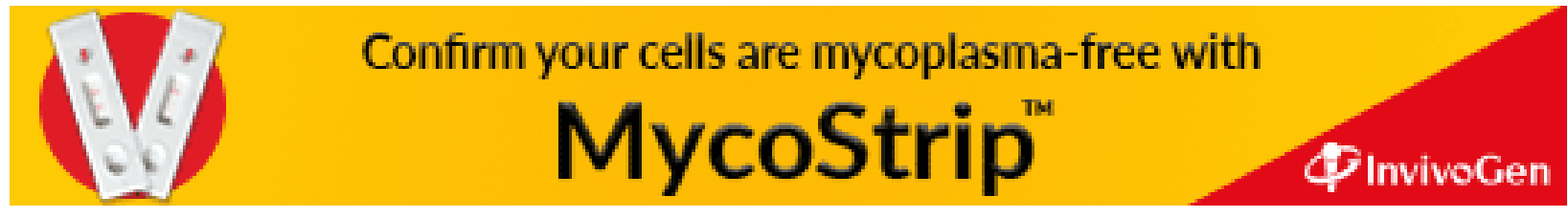

